Textures and Microstructures, 1988, Vols. 8 \& 9, pp. 351-379

Reprints available directly from the publisher

Photocopying permitted by license only

(C) 1988 Gordon and Breach Science Publishers Inc.

Printed in the United Kingdom

\title{
Texture Transition, Micro Shear Bands and Heterogeneous Plastic Strain in F.C.C. and B.C.C. Metals in Rolling
}

\author{
M. BERVEILLER, H. BOUAOUINE, N. FAKRI and P. LIPINSKI
}

Laboratoire de Physique et Mécanique des Matériaux, Ile du Saulcy 57045 Metz Cedex, France

(Received September 20, 1987, in final form December 10, 1987)

\section{Dedicated to the memory of Professor Günter Wassermann}

During the plastic deformation of a metallic polycrystal many kinds of heterogeneous deformation are often observed, both on an intergranular scale and inside the grains. The heterogeneities of the plastic intragranular strains are associated with the latent hardening and with internal stresses.

In order to deal with this problem, it is assumed that some regions of simple glide exist inside the grains, separated by plane interfaces. These interfaces are assumed to be habit planes, separating the domain of simple glide and a homogeneous continuous medium, whose deformation is the one imposed on the polycrystal.

The compatibility relations between the domain of simple glide and the imposed deformation, enable for determination of the gliding amplitude, normal to the habit plane, and the rotation of the crystal lattice inside this domain.

In the case of rolling for F.C.C. and B.C.C. metals, we have analyzed the spin of the crystal lattice in the form of a distribution function. We observe sources and sinks which correspond to the stable orientations, shown by the orientation distribution function. The comparison between these calculations and classical data of rolling texture analysis indicates that a certain number of observed components are due to deformation inhomogeneities, as suggested previously.

The comparison of the orientation of the habit planes shows that there exists a definite relation between habit planes and micro shear bands.

KEY WORDS: Model calculations, heterogeneous deformation, simple glide, lattice rotation, rolling deformation, micro shear bands. 


\section{INTRODUCTION}

The inhomogeneity of the plastic strain of metallic polycrystals is conceived here in the sense of a spatial variation of the number and nature of active slip systems. The notion of "deformation mode" is introduced to characterize any local combination of active slip systems.

Several sources of the inhomogeneous deformation of a polycrystal may be distinguished among which the most important are the relative crystallographic misorientation of grains, the interactions between the grain boundaries and glide systems, the field of internal stresses and the latent hardening.

The classical approaches to the polycrystal plasticity such as Taylor model (Taylor, 1938), relaxed Taylor model (Honnef and Mecking, 1978) or the self-consistent scheme (Kröner, 1961; Berveiller and Zaoui, 1979) describe well the intergranular inhomogeneities of the deformation modes. The strain field within each grain is supposed to be homogeneous.

The microscopic observations do not confirm this rough approximation (Jaoul, 1965; Truszkowski and Krol, 1968). The complexity of the deformation modes at the intragranular scale hinders the global analysis of the confronted phenomena. An interesting approach concerning the interactions between the glide systems and grain boundaries was proposed by Rey and Zaoui (1982) for the bicrystal with a plane interface. This approach was also successful in the case of the tricrystal (Mussot, 1983). The extension of the proposed method to the arbitrary polycrystal configuration seems to be rather difficult.

On the other hand, the formation of dislocation cells during cyclic or monotonic loadings (Mughrabi 1983; Winter, 1974; Rasmussen and Pedersen, 1980), constitutes another form of the plastic inhomogeneity. Certain features of the dislocation cells were modeled by introducing the notion of a habit plane (Berveiller and Bouaouine, 1987) in plasticity; the dislocation cell-wall is described using a plane interface separating two regions of different imposed deformation modes. This habit plane does not introduce a long range stress field.

The regions of localized shears, called shear bands, are observed simultaneously at the macroscopic and microscopic scales and constitute another deformation mode. The formation of shear bands 
may be directly correlated with the imposed overall strains (Korbel and Martin, 1986; Korbel et al., 1986, Fargette and Whitwham, 1976). Experimental results on the shear bands creation indicate that the orientation of these bands depends on the imposed overall loading conditions. For the rolled sheets, the bands are oriented at $35^{\circ}$ with respect to the rolling plane and in the case of tension test, the observed angle between the bands and tension axis is nearly equal to $54^{\circ}$ (Korbel and Martin).

From the metallographic point of view, the decrease of the stacking fault energy (SFE), the addition of a secondary chemical element giving solid solutions or the decrease of the deformation temperature (Haasen, 1958; Meissner, 1959) favour the formation of the shear bands. The effects of a decrease of temperature or a rise of the concentration of an element of addition are similar concerning behaviour of single crystal. Figure 1 shows a notable extension of the state 1 (easy glide) expressing the development of an important latent hardening (Haasen, 1958; Meissner, 1959). This latent hardening is also amplified when SFE decreases. Under such conditions the multislip deformation mode becomes more difficult and the plastic inhomogeneities appear as another energetically advantageous deformation mode. Some habit planes separating the regions of different deformation modes can play a role in formation of the microshear bands nuclei.

Otherwise, the studies of the induced deformation textures of various F.C.C. metals and alloys permitted to classify the textures on two groups. First, called copper textures are typical for pure F.C.C. metals and second, called brass-type textures are rather common for F.C.C. alloys. Various simple models were used to explain the transition between these two types of texture. The different micro-mechanisms such as cross slip (Smallman and Green, 1964), partial dislocations slip (Dillamore and Roberts, 1964), anisotropic interactions between dislocations (Franciosi et al., 1980), were proposed. Wassermann (Wassermann, 1963; Wassermann and Heye, 1968) explained this transition using twinning mechanism. His model was partially shaken by Leffers (1969). On the other hand, Leffers proposed to explain the phenomenon with help of the Sachs model (Sachs, 1928). His approach is based on the idea of intense primary slip. The same deformation mechanism is accepted in this paper.

It is well known that the deformation of pure metals such as 


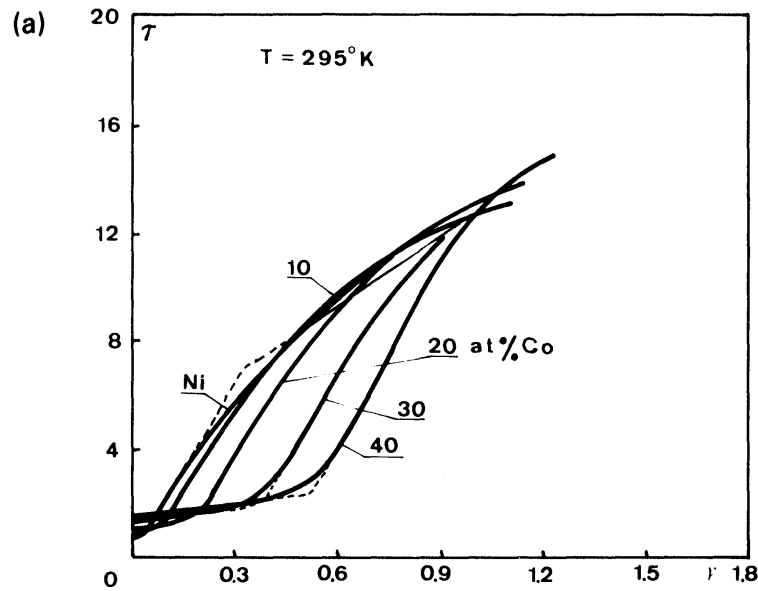

(b)

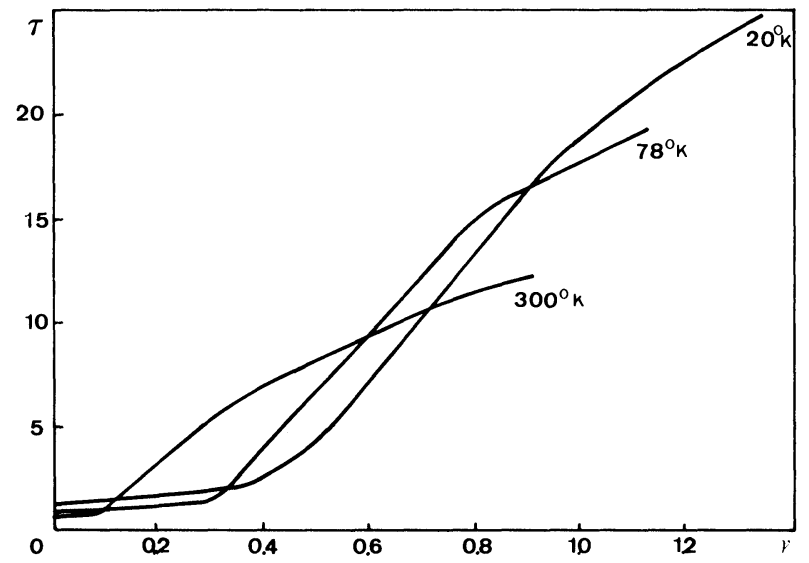

Figure 1 Dependence of the gliding amplitude of stage I for nickel single crystal on: (a) addition elements, according to Hassen (1958), (b) temperature, according to Meissner (1959). $\tau$ and $\gamma$ are respectively critical shear stress and plastic shear.

copper or aluminum occurs rather in the multislip manner. The development of textures of such metals are well described by the classical polycrystal model based on the assumption of the homogeneous intragranular deformation. In the case of metals exhibiting the deformation texture of the brass-type, i.e. for metals charac- 
terized by a weak SFE, solid solutions or when the temperature of deformation is sufficiently low, it is supposed that some texture components are due to inhomogeneous deformations caused by the important latent hardening.

In this paper we propose a model describing certain features of the microstructure of the polycrystal and deformation textures which are linked to a particular aspect of the intragranular plastic inhomogeneities. We suppose that the polycrystal may be deformed by two modes (see Figure 2):

-homogeneous multislip. It occurs especially near the grain boundaries where the internal stresses can only be relaxed by this type of deformation mode;

-single slip. It is facilitated by the strong latent hardening which renders the activation of another systems difficult. Some characteristics of the zone of single glide are determined by relaxing the internal stresses without introducing the multislip mode.

More exactly, we seek a multiple plastic strain field composed of two regions of constant strains and different deformation modes. The regions are supposed to be separated by a plane interface being a habit plane. In this way a compatible and inhomogeneous field of plastic strain is obtained. The orientation of the interface as well as the amplitude of the plastic slip inside the regions of single slip are determined from the general relations of compatibility established by Kröner (1958).

The model has been applied to the rolling of F.C.C. and B.C.C. metals. The directions of the normal vectors to the habit planes and the rotations of the crystal lattices of the zones of single slip have been determined for the both cases. The obtained results are compared respectively with the shear microbands and deformation textures, measured experimentally for the case of the metals and alloys characterized by the strong latent hardening.

\section{THEORY OF INHOMOGENEOUS AND COMPATIBLE PLASTIC DEFORMATION BY SINGLE SLIP}

We suppose that a macrohomogeneous volume element of the polycrystal is composed of $N$ grain families $I$ with the corresponding 
volume fraction $F_{I}$. We formulate this problem throughout the strain rate field $\dot{\varepsilon}_{i j}^{p}(r)$ which is assumed to be inhomogeneous both inside and between grains. The elastic behavior is supposed to be homogeneous and isotropic. Then, the overall plastic strain rate $\dot{E}_{i j}^{p}$ is related to the field $\dot{\varepsilon}_{i j}^{p}(r)$ by

$$
\dot{E}_{i j}^{p}=\frac{1}{V} \int_{V} \dot{\varepsilon}_{i j}^{p}(r) d V
$$

or, in a discrete form

$$
\dot{E}_{i j}^{p}=\sum_{I} F_{I} \dot{\dot{\varepsilon}}_{i j}^{p I}
$$

where $\dot{\bar{\varepsilon}}_{i j}^{p I}$ denotes the mean strain rate value for the grain $I$.

Using the same procedure inside the grain for $n_{I}$ deformation modes, we obtain

$$
\dot{\bar{\varepsilon}}_{i j}^{p I}=\sum f_{\alpha}^{I} \dot{\varepsilon}_{i j}^{p I \alpha}
$$

where $f_{\alpha}^{I}=V_{\alpha}^{I} / V_{I}$ is the volume fraction of $\alpha$ mode inside the grain I. Substituting (3) into (2), one has

$$
\dot{E}_{i j}^{p}=\sum_{I} F_{I} \sum_{\alpha} f_{\alpha}^{I} \dot{\varepsilon}_{i j}^{p I \alpha}
$$

The local plastic strain rate $\dot{\varepsilon}_{i j}^{p l \alpha}$ may be caused by one or more slip rates $\dot{\gamma}^{h}$ on corresponding slip systems defined by the orientation tensor $R_{i j}^{\alpha h}$

$$
R_{i j}^{\alpha h}=\frac{1}{2}\left(m_{i}^{\alpha h} n_{j}^{\alpha h}+m_{j}^{\alpha h} n_{i}^{\alpha h}\right)
$$

such that

$$
\dot{\varepsilon}_{i j}^{p I \alpha}=\sum_{h} R_{i j}^{\alpha h} \dot{\gamma}^{\alpha h}
$$

As usual, the $\vec{m}$ and $\vec{n}$ vectors are the gliding direction and normal to the slip plane, respectively.

From (4) and (6), one obtains

$$
\dot{E}_{i j}^{p}=\sum_{I} F^{I} \sum_{\alpha} f_{\alpha}^{I} \sum_{h} R_{i j}^{h \alpha} \dot{\gamma}^{\alpha h}
$$

Expression (7) describes simultaneously the intra- and inter granular inhomogeneities of the plastic strain rate. This relation is difficult to use for a general kind of aggregate because of the complex form 
of constitutive relations for plastic behavior. We restrict ourselves to metals with high latent hardening for which it is assumed that only one single slip deformation mode is active in a part of a grain.

Moreover, it is supposed that the complementary part of the grain undergoes a multislip deformation mode, like the Taylor mechanism. This last one may exist near grain boundaries or triple nodes where internal stresses are important (see Figure 2). Following Taylor, we suppose that the strain rate in the multislip deformation zone is equal to the overall one $\dot{E}_{i j}^{p}$, independently of the grain orientation.

Thus, decomposition (7) may be rewritten

$$
\dot{E}_{i j}^{p}=\sum_{I} f^{I} R_{i j}^{I h} \dot{\gamma}^{h}+f \dot{E}_{i j}^{p}
$$

with

$$
\sum_{I} f^{I}+f=1
$$

$f$ is now the volume fraction of the polycrystal which undergoes the uniform strain rate $\dot{E}_{i j}^{p}$ by multislip and $f^{I}$ means the volume of single slip zone inside the grain $I$.

Now we can conclude that the average strain rate over a given grain is, of course, different from the applied macroscopic deformation, since

$$
\frac{1}{F_{I}}\left\{f^{I} R_{i j}^{I h} \dot{\gamma}^{h}+\left(F^{I}-f^{I}\right) \dot{E}_{i j}^{p}\right\} \neq \dot{E}_{i j}^{p}
$$

It is obvious that the plastic strain rate is inhomogeneous inside the grains, equal to $R_{i j}^{I k} \dot{\gamma}^{k}$ within the single slip zone and $\dot{E}_{i j}^{p}$ outside. Neglecting the interactions between the various single slip regions, one has to find for each grain, a single slip zone, located in a matrix with homogeneous plastic strain rate $\dot{E}_{i j}^{p}$. The shape and the boundaries of this zone must be determined to assure the compatibility of the strain rate field. The solution of this problem, which is discussed by Lipinski et al., shows that it is possible to obtain such a field (piecewise constant) for a penny shape volume.

In this case, the study of the compatibility problem can be reduced to the analysis of the compatibility conditions through the interface between single slip zone and matrix.

Following the plastic strain compatibility conditions developed by Kröner (1958), these conditions are written down (Berveiller and 
Bouaouine, 1987)

$$
\varepsilon_{i k m} \varepsilon_{j l n}\left(\dot{\varepsilon}_{m n}^{p I}-\dot{E}_{m n}^{p}\right) N_{k} N_{l}=0
$$

where $\varepsilon_{i j k}$ is the permutation tensor and $N_{j}$ the normal unit vector to the interface separating the two deformation modes.

Using the property (6), the following holds

$$
\varepsilon_{i k m} \varepsilon_{j l n}\left(R_{m n}^{I h} \dot{\gamma}^{h}-\dot{E}_{m n}^{p}\right) N_{k} N_{l}=0
$$

We denote: $\left(\dot{E}_{l j}^{p}-R_{i j}^{I h} \dot{\gamma}^{h}\right)=\Delta \dot{\varepsilon}_{i j}^{p I}$.

It can be easily verified (Berveiller, 1980) that system (12) has a non-trivial solution only if at least one eigenvalue of the tensor $\Delta \dot{\varepsilon}_{i j}^{p I}$ vanishes. Applying the properties of the third scalar invariant of symmetric tensors, the above condition is equivalent to:

$$
\operatorname{Det}\left(\Delta \dot{\varepsilon}_{i j}^{p I}\right)=0
$$

If Eq. (13) is verified, it means if the strain tensor in the region of single slip is such that $\operatorname{Det}\left(\Delta \dot{\varepsilon}_{i j}^{p I}\right)=0$, the normal vector to the interface between the two zones is obtained from relations (12) which takes the explicit form:

$$
\begin{aligned}
& N_{2}^{2} \Delta \dot{\varepsilon}_{33}^{p I}+N_{3}^{2} \Delta \dot{\varepsilon}_{22}^{p I}-2 N_{2} N_{3} \Delta \dot{\varepsilon}_{23}^{p I}=0 \\
& N_{1}^{2} \Delta \dot{\varepsilon}_{33}^{p I}+N_{3}^{2} \Delta \dot{\varepsilon}_{11}^{p I}-2 N_{1} N_{2} \Delta \dot{\varepsilon}_{13}^{p I}=0 \\
& N_{1}^{2} \Delta \dot{\varepsilon}_{22}^{p I}+N_{2}^{2} \Delta \dot{\varepsilon}_{11}^{p I}-2 N_{1} N_{2} \Delta \dot{\varepsilon}_{12}^{p I}=0 \\
& N_{3}^{2} \Delta \dot{\varepsilon}_{12}^{p I}-N_{1} N_{3} \Delta \dot{\varepsilon}_{23}^{p I}-N_{2} N_{3} \Delta \dot{\varepsilon}_{13}^{p I}+N_{1} N_{2} \Delta \dot{\varepsilon}_{33}^{p I}=0 \\
& N_{2}^{2} \Delta \dot{\varepsilon}_{13}^{p I}-N_{2} N_{3} \Delta \dot{\varepsilon}_{12}^{p I}-N_{1} N_{2} \Delta \dot{\varepsilon}_{23}^{p I}+N_{1} N_{2} \Delta \dot{\varepsilon}_{22}^{p I}=0 \\
& N_{1}^{2} \Delta \dot{\varepsilon}_{23}^{p I}-N_{1} N_{3} \Delta \dot{\varepsilon}_{12}^{p I}-N_{1} N_{2} \Delta \dot{\varepsilon}_{13}^{p I}+N_{2} N_{3} \Delta \dot{\varepsilon}_{11}^{p I}=0 .
\end{aligned}
$$

Generally, the plastic strain at the zone of single slip is accompanied by a plastic spin $\dot{\omega}_{i j}^{p}$ due to plastic slip. This plastic rotation induces an elastic spin $\dot{\omega}_{i j}^{e}$ of the crystal lattice. This last one is determined from the first order compatibility condition (Kröner, 1958). Because $\dot{\varepsilon}_{i j}^{e}(r)$ is constant, the first order compatibility condition takes the form:

$$
\operatorname{Curl}\left[\dot{\varepsilon}^{p}(r)+\dot{\omega}^{p}(r)+\dot{\omega}^{e}(r)\right]=0
$$

The above relation may be written as:

$$
\left(\dot{\omega}_{i j}^{e}+\dot{\omega}_{i j}^{p}\right)_{, k}=\dot{\varepsilon}_{k i, j}^{p}-\dot{\varepsilon}_{k j, i}^{p}
$$


or, in this case

$$
-\left(\dot{\omega}_{i j}^{e I}+\dot{\omega}_{i j}^{p I}\right) N_{k}=\Delta \dot{\varepsilon}_{k i}^{p I} N_{j}-\Delta \dot{\varepsilon}_{k j}^{p} N_{i}
$$

where it is assumed that the overall total spin $\dot{\Omega}_{i j}^{T}$ disappears $\left(\dot{\Omega}_{i j}^{T}=0\right)$ in the matrix as it is the case for all axisymmetric (tension, compression, extrusion) or plane (rolling) tests.

The elastic spin $\dot{\omega}_{i j}^{e l}$ inside the single slip zone now becomes

$$
\dot{\omega}_{i j}^{e I} N_{k}=\Delta \dot{\varepsilon}_{k j}^{p} N_{i}-\Delta \dot{\varepsilon}_{k i}^{p} N_{j}-\dot{\omega}_{i j}^{p I} N_{k}
$$

Knowing the overall plastic strain rate $\dot{E}_{i j}^{p}$ and the active system at the single slip zone, $\Delta \dot{\varepsilon}_{i j}^{p}$ and $\dot{\omega}_{i j}^{p}$ can be calculated, the normal vector $N_{i}$ may be determined from (14) and finally the components of $\dot{\omega}_{i j}^{e I}$ may be found using (18). This elastic rotation tensor defines the crystal lattice rotation of the single slip zones.

If the crystallographic orientations of lattices are given by the Euler angles $\varphi_{1}, \Phi, \varphi_{2}$ introduced by Bunge (1969), the rotation field evolution (texture) is determined as a function of the rates $\dot{\varphi}_{1}$, $\dot{\Phi}$ and $\dot{\varphi}_{2}$

$$
\begin{aligned}
& \dot{\varphi}_{1}=-\frac{\sin \varphi_{2}}{\sin \Phi} \dot{\omega}_{23}^{e I}+\frac{\cos \varphi_{2}}{\sin \Phi} \dot{\omega}_{13}^{e I} \\
& \dot{\Phi}=-\sin \varphi_{2} \dot{\omega}_{13}^{e I}-\cos \varphi_{2} \dot{\omega}_{23}^{e I} \\
& \dot{\varphi}_{2}=-\dot{\omega}_{12}^{e I}-\frac{1}{\cos \Phi} \dot{\varphi}_{1}
\end{aligned}
$$

The interface separating the zone of single slip and the zone of multi-slip constitutes in fact a habit plane. The notion of such a plane is commonly used to describe twinning or martensitic transformations. This concept was extended by Berveiller and Bouaouine (1987) to the case of the plasticity of single crystals and successfully applied to explain some features of dislocation cell-walls. In this paper the components of the normal vector to the habit plane are determined from (14) and represent the normal vector at the initial configuration of the material. Experimentally, the interfaces are observed at the current configuration (after the material was transformed). In order to compare the theoretical results with the experimental measures, it is necessary to transform the obtained vector to the current configuration using the classical rules of the finite strain theory. 
The theoretical framework presented up to now is quite general and may be applied to any situation of two piecewise constant deformation modes (both multislip) separated by the habit plane. In the following, in order to take into consideration the latent hardening of the F.C.C. metals, we specialize the obtained expressions for a single slip mode. In fact, the latent hardening increases sufficiently the critical shear stresses on the other glide systems and simultaneously the internal stresses associated with the primary glide are partially or even totally relaxed by the formation of the habit plane.

In this case, denoting by $\vec{n}$ the normal vector to the slip plane and by $\vec{m}$ the slip direction the usual relations for plastic strains and plastic rotations holds:

$$
\begin{aligned}
\dot{\varepsilon}_{i j}^{p I} & =\frac{1}{2}\left(m_{i} n_{j}+m_{j} n_{i}\right) \gamma=R_{i j} \dot{\gamma} \\
\dot{\omega}_{i j}^{p I}=\frac{1}{2}\left(m_{i} n_{j}-m_{j} n_{i}\right) \gamma & =S_{i j} \dot{\gamma}
\end{aligned}
$$

where $\dot{\gamma}$ is the slip rate of the considered active system. Now, for an arbitrary overall strain $\dot{E}_{i j}^{p}$, compatibility condition (13) takes the form of the third degree equation for $\dot{\gamma}$.

$$
\begin{aligned}
& A \dot{\gamma}^{3}-B \dot{\gamma}^{2}+C \dot{\gamma}-D=0 \\
& A= R_{11} R_{22} R_{33}+2 R_{12} R_{13} R_{23}-R_{22} R_{13}^{2} \\
&-R_{11} R_{23}^{2}-R_{33} R_{12}^{2}=\operatorname{Det}\left(R_{1 j}\right) \\
& B= R_{11} R_{22} \dot{E}_{33}^{p}+R_{11} R_{33} \dot{E}_{22}^{p}+R_{22} R_{33} \dot{E}_{11}^{p} \\
&+2 R_{12} R_{23} \dot{E}_{13}^{p}+2 R_{12} R_{13} \dot{E}_{23}^{p} \\
&+2 R_{13} R_{23} \dot{E}_{12}^{p}-2 R_{13} R_{22} \dot{E}_{13}^{p}-R_{13}^{2} \dot{E}_{22}^{p}-2 R_{11} R_{23} \dot{E}_{23}^{p} \\
&-R_{23}^{2} \dot{E}_{11}^{p}-2 R_{12} R_{33} \dot{E}_{12}^{p}-R_{12}^{2} \dot{E}_{33}^{p} \\
& C= R_{11} \dot{E}_{22}^{p} \dot{E}_{33}^{p}+R_{22} \dot{E}_{11}^{p} \dot{E}_{33}^{p}+R_{33} \dot{E}_{11}^{p} \dot{E}_{22}^{p} \\
&+2 R_{12} \dot{E}_{13}^{p} \dot{E}_{23}^{p}+2 R_{23} \dot{E}_{12}^{p} \dot{E}_{13}^{p} \\
&+2 R_{13} \dot{E}_{12}^{p} \dot{E}_{23}^{p}-R_{22} \dot{E}_{13}^{p} \dot{E}_{13}^{p}-2 R_{13} \dot{E}_{22}^{p} \dot{E}_{13}^{p}-R_{11} \dot{E}_{23}^{p} \dot{E}_{23}^{p} \\
&-2 R_{23} \dot{E}_{11}^{p} \dot{E}_{23}^{p}-R_{33} \dot{E}_{12}^{p} \dot{E}_{12}^{p}-2 R_{12} \dot{E}_{12}^{p} \dot{E}_{33}^{p} \\
& D= \dot{E}_{11}^{p} \dot{E}_{22}^{p} \dot{E}_{33}^{p}+2 \dot{E}_{12}^{p} \dot{E}_{23}^{p} \dot{E}_{13}^{p}-\dot{E}_{22}^{p} \dot{E}_{13}^{p} \dot{E}_{13}^{p} \\
&-\dot{E}_{11}^{p} \dot{E}_{23}^{p} \dot{E}_{23}^{p}-\dot{E}_{33}^{p} \dot{E}_{12}^{p} \dot{E}_{12}^{p}=\operatorname{Det}\left(\dot{E}_{i j}^{p}\right)
\end{aligned}
$$

For usual metals, the normal vector to the slip planes is orthogonal to the glide direction and the following relation is satisfied

$$
A=\operatorname{Det}\left(R_{i j}\right)=0
$$


Under such condition, relation (21) becomes a second degree equation the form of which may be still simplified for particular forms of $\dot{E}_{i j}^{p}$ tensor. The case of rolling tests is analysed at chapter III.

In order to render the theory more applicable, we propose a solution of a double indetermination problem associated with the symmetry of the F.C.C. crystal and the form of Eqs. (14).

For metals possessing the cubic symmetry, there exists twelve glide systems which are equivalent from the crystallographic view point. It means that kinematic conditions (14) and (21) may be satisfied by twelve equivalent manners. Each solution leads to different interfaces and to different elastic rotations of the lattice. This degeneracy problem of kinematic type may be solved using the energetic criterion similar to that proposed by Taylor. A chosen solution, among all kinematically admissible fields, renders minimal the local plastic work. A deeper analysis of this problem is done at appendix I.

The second indetermination is due to the form of Eqs. (14), which for a given value of $\dot{\gamma}$ or $\Delta \dot{\varepsilon}_{i j}^{p I}$ leads to two distinct solutions for the normal vector, says $\vec{N}_{1}$ and $\vec{N}_{2}$. It is easy to verify that these vectors are orthogonal and they define two orthogonal habit planes. The lattice rotations of the single slip zone are strictly related to the normal vector of the habit plane. So, there are also two solutions for the lattice rotations.

This double solution is treated by the intermediary of a relative stability criterion which allows to classify two different rotation modes. This criterion, based on the comparison of elastic energies associated with partially compatible configurations, is discussed in the Appendix II. The application of the model is presented in the following chapters.

\section{APPLICATION TO THE ROLLING OF F.C.C. METALS}

In the case of the simulation of rolling, the overall plastic strain tensor takes the form:

$$
\dot{E}_{i j}^{p}=\dot{E}^{p}\left[\begin{array}{rrr}
1 & 0 & 0 \\
0 & 0 & 0 \\
0 & 0 & -1
\end{array}\right]
$$


Table $1 \quad R$ and $S$ tensors for slip systems of F.C.C. metals

\begin{tabular}{|c|c|c|c|c|c|c|c|c|}
\hline & \multicolumn{2}{|l|}{ Slip system } & \multicolumn{3}{|c|}{$\begin{array}{l}R_{i j} \text { tensor } \\
\times 1 / 2 \sqrt{6}\end{array}$} & \multicolumn{3}{|c|}{$\begin{array}{l}S_{i j} \text { tensor } \\
\times 1 / 2 \sqrt{6}\end{array}$} \\
\hline $\mathrm{A} 2$ & $\begin{array}{l}\text { normale } \\
\left(\begin{array}{lll}1 & 1 & 1\end{array}\right)\end{array}$ & $\begin{array}{l}\text { direction } \\
{\left[\begin{array}{lll}0 & \overline{1} & 1\end{array}\right]}\end{array}$ & 0 & $\begin{array}{r}1 \\
-2\end{array}$ & $\begin{array}{r}-1 \\
0 \\
2\end{array}$ & $\begin{array}{r}0 \\
1 \\
-1\end{array}$ & $\begin{array}{r}-1 \\
0 \\
2\end{array}$ & $\begin{array}{r}1 \\
-2 \\
0\end{array}$ \\
\hline A3 & $\left(\begin{array}{lll}\overline{1} & 1 & 1\end{array}\right)$ & {$\left[\begin{array}{lll}1 & 0 & 1\end{array}\right]$} & -2 & $\begin{array}{l}1 \\
0\end{array}$ & $\begin{array}{l}0 \\
1 \\
2\end{array}$ & 0 & $\begin{array}{l}1 \\
0\end{array}$ & $\begin{array}{r}2 \\
-1 \\
0\end{array}$ \\
\hline A6 & $\left(\begin{array}{lll}\overline{1} & 1 & 1\end{array}\right)$ & {$\left[\begin{array}{lll}1 & 1 & 0\end{array}\right]$} & -2 & $\begin{array}{l}0 \\
2\end{array}$ & $\begin{array}{l}1 \\
1 \\
0\end{array}$ & 0 & $\begin{array}{l}2 \\
0\end{array}$ & $\begin{array}{l}1 \\
1 \\
0\end{array}$ \\
\hline B2 & $\left(\begin{array}{lll}1 & 1 & 1\end{array}\right)$ & {$\left[\begin{array}{lll}0 & 1 & 1\end{array}\right]$} & 0 & $\begin{array}{l}-1 \\
-2\end{array}$ & $\begin{array}{l}1 \\
0 \\
2\end{array}$ & 0 & $\begin{array}{l}1 \\
0\end{array}$ & $\begin{array}{r}-1 \\
-2 \\
0\end{array}$ \\
\hline B4 & $\left(\begin{array}{lll}1 & 1 & 1\end{array}\right)$ & {$\left[\begin{array}{lll}\overline{1} & 0 & 1\end{array}\right]$} & -2 & $\begin{array}{r}-1 \\
0\end{array}$ & $\begin{array}{l}0 \\
1 \\
2\end{array}$ & & $\begin{array}{r}-1 \\
0\end{array}$ & $\begin{array}{r}-2 \\
-1 \\
0\end{array}$ \\
\hline B5 & $\left(\begin{array}{lll}1 & 1 & 1\end{array}\right)$ & {$\left[\begin{array}{lll}1 & 1 & 0\end{array}\right]$} & 2 & $\begin{array}{r}0 \\
-2\end{array}$ & $\begin{array}{r}1 \\
-1 \\
0\end{array}$ & 0 & & $\begin{array}{r}1 \\
-1 \\
0\end{array}$ \\
\hline $\mathrm{C} 1$ & $\left(\begin{array}{lll}1 & 1 & 1\end{array}\right)$ & {$\left[\begin{array}{lll}0 & 1 & 1\end{array}\right]$} & 0 & $\begin{array}{l}1 \\
2\end{array}$ & $\begin{array}{r}1 \\
0 \\
-2\end{array}$ & & $\begin{array}{r}-1 \\
0\end{array}$ & $\begin{array}{r}-1 \\
-2 \\
0\end{array}$ \\
\hline $\mathrm{C} 3$ & $\left(\begin{array}{lll}1 & 1 & 1\end{array}\right)$ & {$\left[\begin{array}{lll}1 & 0 & 1\end{array}\right]$} & 2 & $\begin{array}{l}1 \\
0\end{array}$ & $\begin{array}{r}0 \\
1 \\
-2\end{array}$ & 0 & $\begin{array}{l}1 \\
0\end{array}$ & $\begin{array}{r}-2 \\
-1 \\
0\end{array}$ \\
\hline C5 & $\left(\begin{array}{lll}1 & 1 & \overline{1}\end{array}\right)$ & {$\left[\begin{array}{lll}1 & 1 & 0\end{array}\right]$} & 2 & $\begin{array}{r}0 \\
-2\end{array}$ & $\begin{array}{r}-1 \\
1 \\
0\end{array}$ & 0 & & $\begin{array}{r}-1 \\
1 \\
0\end{array}$ \\
\hline D1 & $\left(\begin{array}{lll}1 & \overline{1} & 1\end{array}\right)$ & {$\left[\begin{array}{lll}0 & 1 & 1\end{array}\right]$} & 0 & $\begin{array}{r}1 \\
-2\end{array}$ & $\begin{array}{l}1 \\
0 \\
2\end{array}$ & & $\begin{array}{r}-1 \\
0\end{array}$ & $\begin{array}{r}-1 \\
2 \\
0\end{array}$ \\
\hline D4 & $\left(\begin{array}{lll}1 & 1 & 1\end{array}\right)$ & {$\left[\begin{array}{lll}\overline{1} & 0 & 1\end{array}\right]$} & & $\begin{array}{l}1 \\
0\end{array}$ & $\begin{array}{r}0 \\
-1 \\
2\end{array}$ & 0 & & $\begin{array}{r}-2 \\
1 \\
0\end{array}$ \\
\hline D6 & $\left(\begin{array}{lll}1 & 1 & 1\end{array}\right)$ & {$\left[\begin{array}{lll}1 & 1 & 0\end{array}\right]$} & 2 & $\begin{array}{r}0 \\
-2\end{array}$ & $\begin{array}{l}1 \\
1 \\
0\end{array}$ & & $\begin{array}{r}-2 \\
0\end{array}$ & $\begin{array}{l}1 \\
1 \\
0\end{array}$ \\
\hline
\end{tabular}


The coefficient $D=\operatorname{det}\left(\dot{E}_{i j}^{p}\right)$ of relation (22) disappears and Eq. (21) may be rewritten as:

$$
\dot{\gamma}(B \dot{\gamma}-C)=0
$$

The non-trivial solution of $(25)(\dot{\gamma} \neq 0)$ for any slip system is given by

$$
\dot{\gamma}=\frac{C}{B}=\dot{E}^{p} \frac{R_{22}}{\left(R_{11} R_{22}-R_{12}^{2}\right)-\left(R_{22} R_{33}-R_{23}^{2}\right)}
$$

The F.C.C. metals are characterized by $R$ and $S$ tensors of twelve easy glide systems of $\{111\}\langle 110\rangle$ type. The components of $R$ and $S$ on the coordinate system of the crystal lattice (axis $\langle 100\rangle$ ) are given in Table 1. The orientation of grains of the polycrystal with respect to the macroscopic coordinate system, in which strain tensor is expressed, is defined by three Euler angles $\varphi_{1}, \Phi, \varphi_{2}$ introduced by Bunge (1969).

The numerical calculations have been performed for the value of $\dot{E}^{p}=0.1$ and orientation angles $\varphi_{1}, \Phi, \varphi_{2}$ have been varying from $-20^{\circ}$ to $110^{\circ}$ with step of $5^{\circ}$. First, the slip amplitude is determined which satisfies Eq. (26). Next, the normal vectors to the habit planes at the current configuration are calculated.

Finally, the rotation field of crystal lattice is deduced and presented in a form of sections of Euler angle space by planes of $\varphi_{2}=$ const.

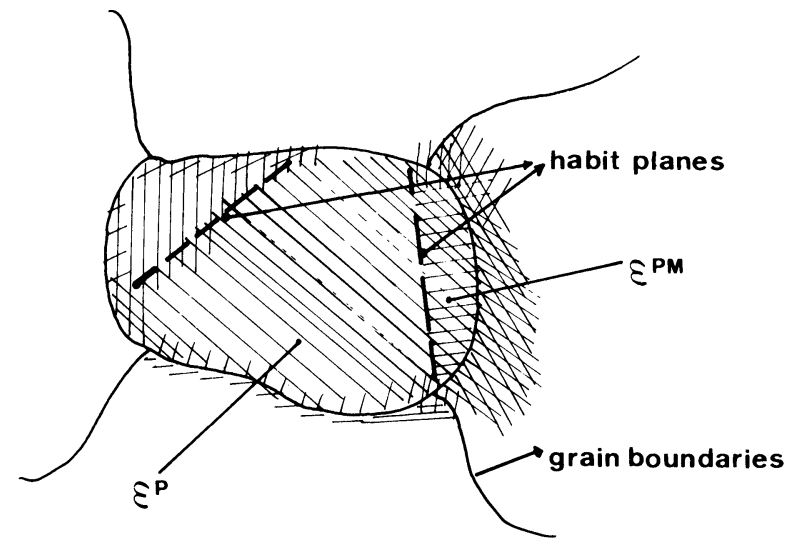

Figure 2. Schematic representation of inhomogeneous multislip inside a grain of polycrystal. 

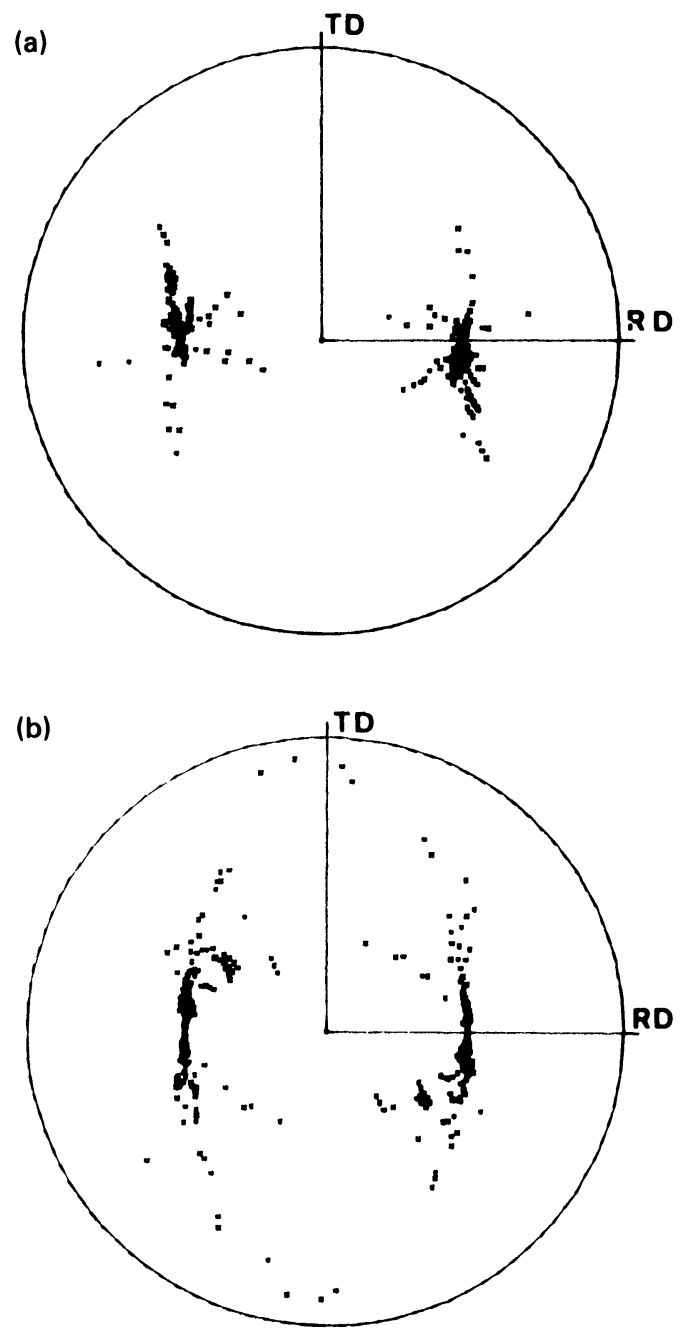

Figure 3. Representation of the normal vectors to the habit planes for various orientations of $\varphi_{2}$ and $\Phi\left(\varphi_{1}=30^{\circ}\right)$ for F.C.C. and B.C.C. metals. (a) higher stability criterion, (b) lower stability criterion. 


\section{III.1 Normal vectors to habit planes}

The stereographic projection of normal vectors to habit planes, calculated using expression (14), in the (RD, TD) plane is presented in Figure 3. The vectors obtained with help of expression (14) are transformed to the current configuration using the classical rules of the finite strain theory. Only some orientations defined by the following intervals of angles are presented in this figure.

$$
\begin{aligned}
\varphi_{1} & =30^{\circ} \\
0 & <\varphi_{2}<90^{\circ} \quad\left(\text { with } \Delta \varphi_{2}=5^{\circ}\right) \\
0 & <\Phi<90^{\circ} \quad\left(\text { with } \Delta \Phi=5^{\circ}\right)
\end{aligned}
$$

Results for another value of $\varphi_{1}$ are analogous. Figure $3 \mathrm{a}$ corresponds to the habit planes of higher stability (see Appendix II). In Figure $3 b$ are plotted the orientations of normal vectors to the habit planes of reduced stability. A more important scatter of the normal vectors can be observed in the second case, but similar distribution is observed for both solutions. The essential conclusions are that the habit planes are parallel to the transverse direction of rolling (TD), the repartition of the vector projections is not random, but may be approached by two ideal components symmetric with respect to the (ND, TD) plane and situated at about $38^{\circ}$ to the rolling direction (RD) on the (RD, ND) plane. When presented in the initial configuration, this angle is about $45^{\circ}$. The obtained ideal orientation of habit planes is very close to that of microshear bands usually observed after rolling of F.C.C. metals. The agreement becomes very good especially when compared with experimental results obtained after rolling at low temperature or for the case of solid solutions for which the latent hardening is relatively important. The last remark may be reformulated in another way: it exists a relation of cause-effect between the inhomogeneous multislip described in this paper and the formation of microshear bands.

\section{III.2 Field of crystallographic lattice rotations}

The rotation field is presented in a discrete manner. Various sections of the Euler angle space by $\varphi_{2}=$ const plane are plotted. 


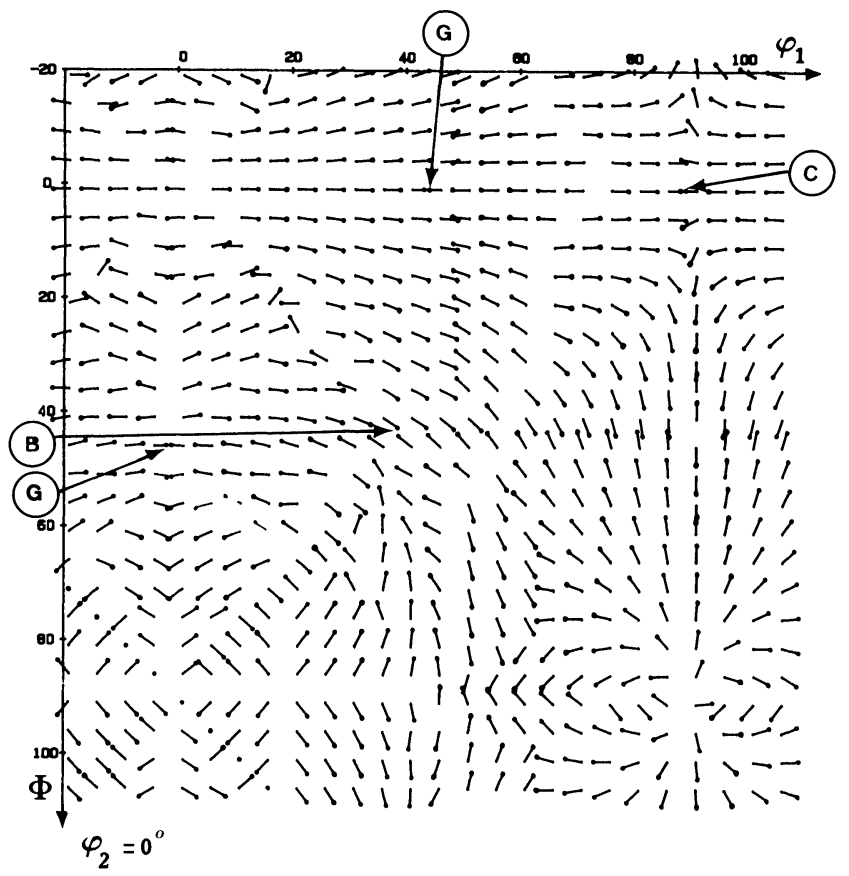

Figure 4 Rotation field of F.C.C. crystalline lattices in $\varphi_{2}=0$ plane using higher stability criterion, and experimental texture components according to $A$ (Hirsch et al., (1981) and $A$ (Van Houtte, 1984).

The variations of $\varphi_{1}, \Phi, \varphi_{2}$ angles are presented with help of oriented arrows calculated for $\dot{E}^{p}=0.1$.

In order to render the figure more legible the double solution of the problem was separated into two using of the stability criterion discussed in Appendix III.

It is obvious that the complete solution of the rotation problem is composed of the (multiple) Taylor rotation field for the part of grains deformed by $\dot{E}_{i j}^{p}$ and by the double field associated with single slip mode. Here, we only present the second one.

Figures 4, 5, 6, 7 present different sections of rotation field by planes of $\varphi_{2}=$ const. The sources and sinks are visible on these figures. These last ones correspond to the stable orientations, which must be observed among the texture components. In the same 


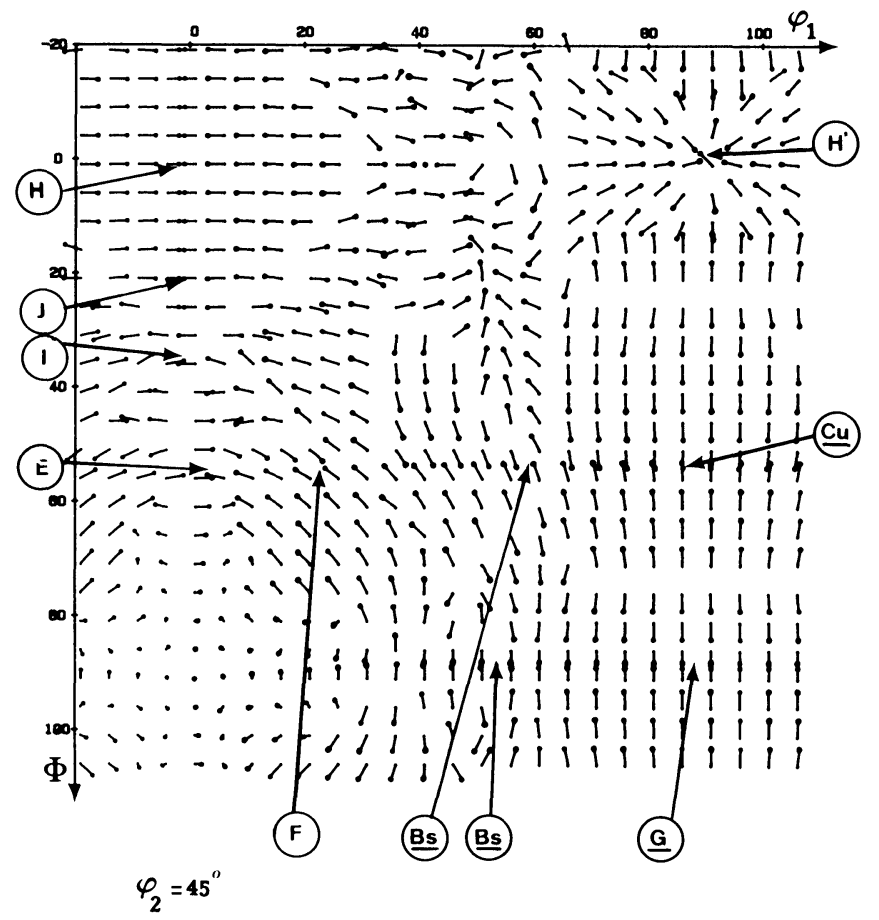

Figure 5 Rotation field of F.C.C. crystalline lattices in $\varphi_{2}=45^{\circ}$ plane using higher stability criterion, and experimental texture components according to $A$ (Hirsch et al., 1981) and $A$ (Van Houtte 1984).

figures the various rolling texture components of F.C.C. metals are presented. Table 2 summarizes the experimental results by Van Houtte (1984), and Hirsch, Virnich and Lücke (1981). The numerous principal components (like $\mathrm{G}$ and BS) which characterize essentially brass-type textures are numerically reproduced.

On the other hand, the applied model does not predict the components $\{112\}\langle 111\rangle(\mathrm{Cu})$ and $\{123\}\langle 634\rangle(\mathrm{S})$ which are often observed for copper or aluminum. It is well documented that the homogeneous multislip deformation mode is dominant for these metals. The presented method was established to model the single slip deformation mode. Then it is not surprising that these texture components are absent in the present results. 


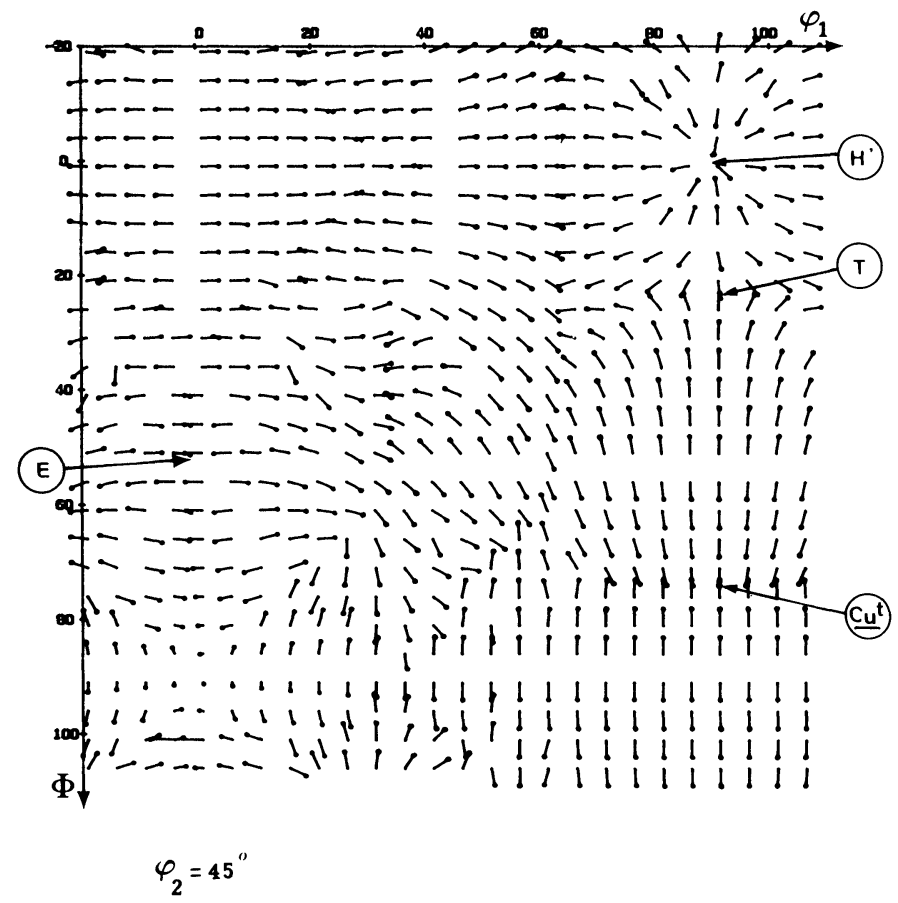

Figure 6 Rotation field of F.C.C. crystalline lattices in $\varphi_{2}=45^{\circ}$ plane using lower stability criterion, and experimental texture components according to $A$ (Hirsch et al., 1981) and $A$ (Van Houtte, 1984).

\section{APPLICATION TO THE ROLLING OF B.C.C. METALS}

Similarly as for F.C.C. metals, the loading of the elementary volume of B.C.C. metals is described by Eq. (24). The solution procedure is analogous to that presented on Chapter III.

The components of $R$ and $S$ tensors on the coordinate system of crystal (axes $\{100\})$ for twelve glide systems $\langle 110\rangle\{111\}$ are given in Table 3.

The obtained results are presented in the similar manner, it means the projection of normal vectors to the habit planes and rotation fields are given. For B.C.C. metals it is obvious to present the orientation distribution function by cutting the Euler angle 


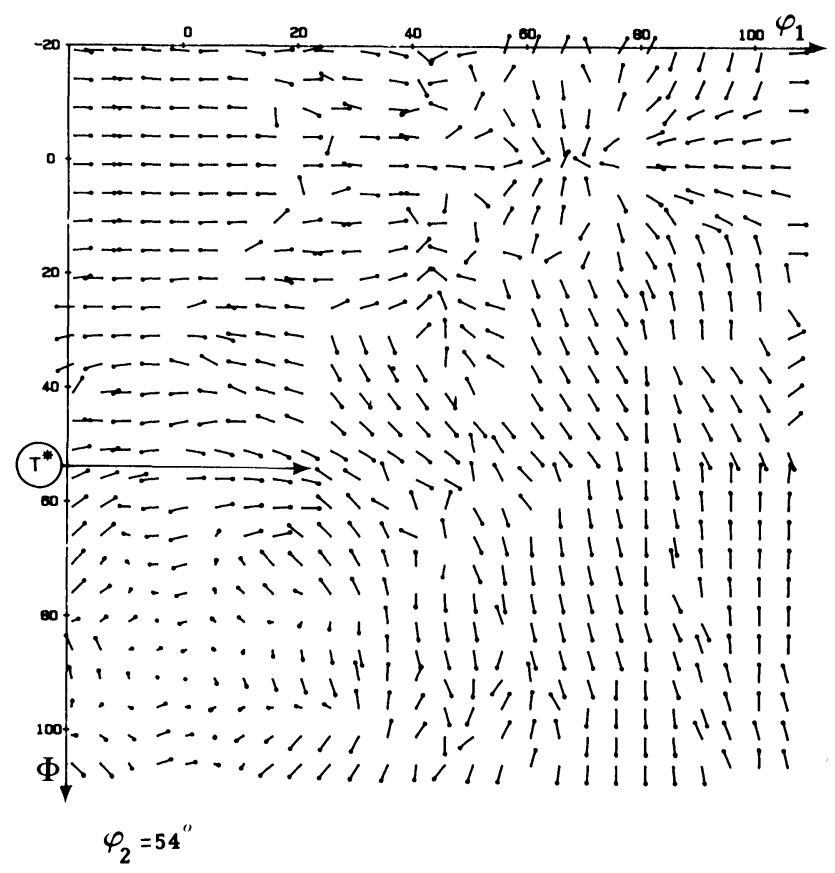

Figure 7 Rotation field of F.C.C. crystalline lattices in $\varphi_{2}=54^{\circ}$ plane using higher stability criterion and experimental texture components according to $\underline{A}$ (Hirsch $e t$ al., 1981) and $A$ (Van Houtte, 1984).

space by planes by $\varphi_{1}=$ const. This type of representation is adopted here.

\section{IV.1 Nornal vectors to habit planes}

The distribution of habit planes orientation with respect to the overall coordinate system is the same as for F.C.C. metals. This result was expected because the slip amplitudes and normal vectors to the habit planes depend on the symmetric tensor $R$ which is the same for F.C.C. and B.C.C. metals.

\section{IV.2 Rotation field of crystalline lattices}

The rotation field is presented in the discrete manner for various sections of the Euler angle space corresponding to various planes 
Table 2 Texture components observed in F.C.C. rolled metals. (a) after J. Hirsch, K. H. Virnich, K. Lücke (1987) (b) after P. Van Houtte (1984)

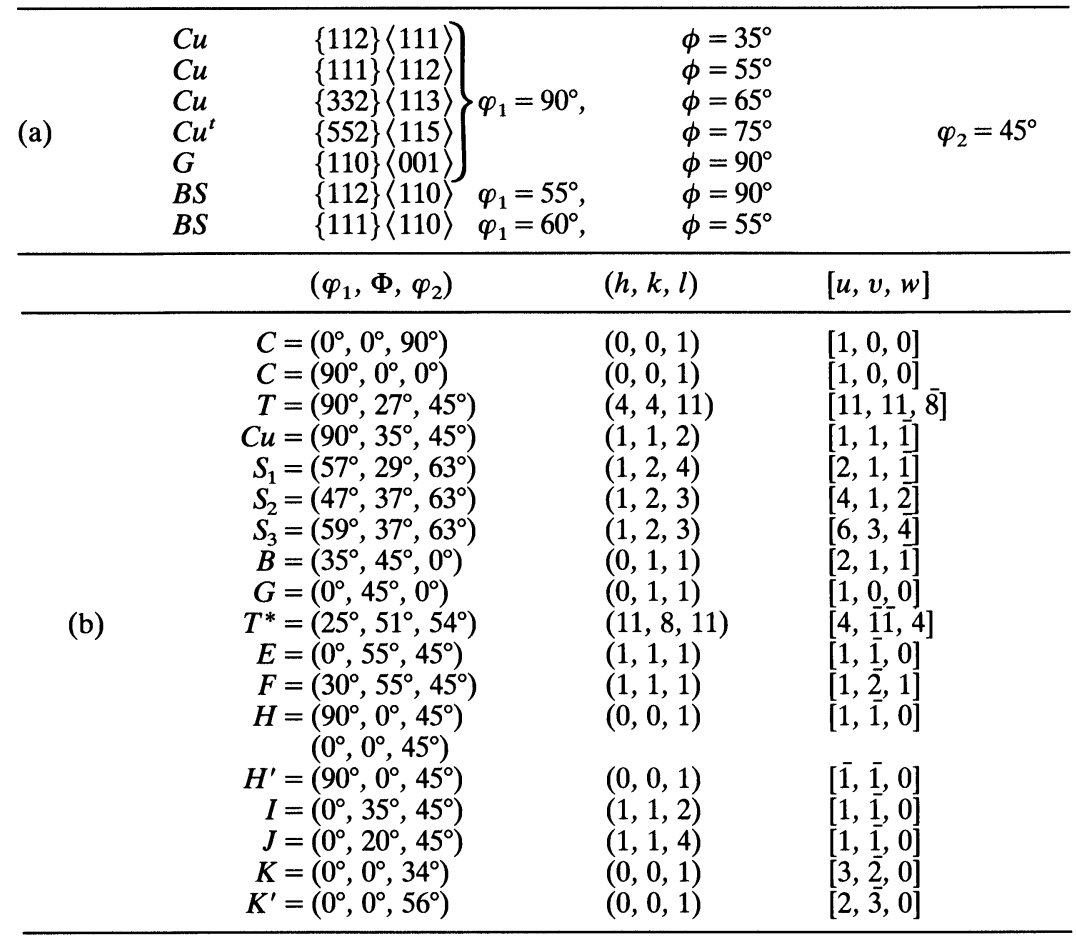

$\varphi_{1}=$ const. The increments $\Delta \varphi_{2}$ and $\Delta \Phi$ of two Euler angles are visualized by arrows in $\varphi_{1}=$ const planes. These increments have been calculated for $\dot{E}^{p}=0.1$.

Figures 8,9 and 10 show three sections of the Euler space for $\varphi_{1}=0^{\circ}, \varphi_{1}=50^{\circ}$, and $\varphi_{1}=90^{\circ}$. Parallelly, the partial experimental results, deduced from the paper of Bunge (1968) and corresponding to the $70 \%$ rolled sheet-steel, are presented in these figures.

As for F.C.C. metals, we may conclude that some components of the experimental textures, which cannot be explained by classical models, are well reproduced by the applied approach, and as a consequence, these components correspond to the inhomogeneous multislip deformation mode. Of course, like in the case of F.C.C. metals, a part of the texture associated with multislip is not present in these figures. 
Table $3 R$ and $S$ tensors for slip systems of B.C.C. metals

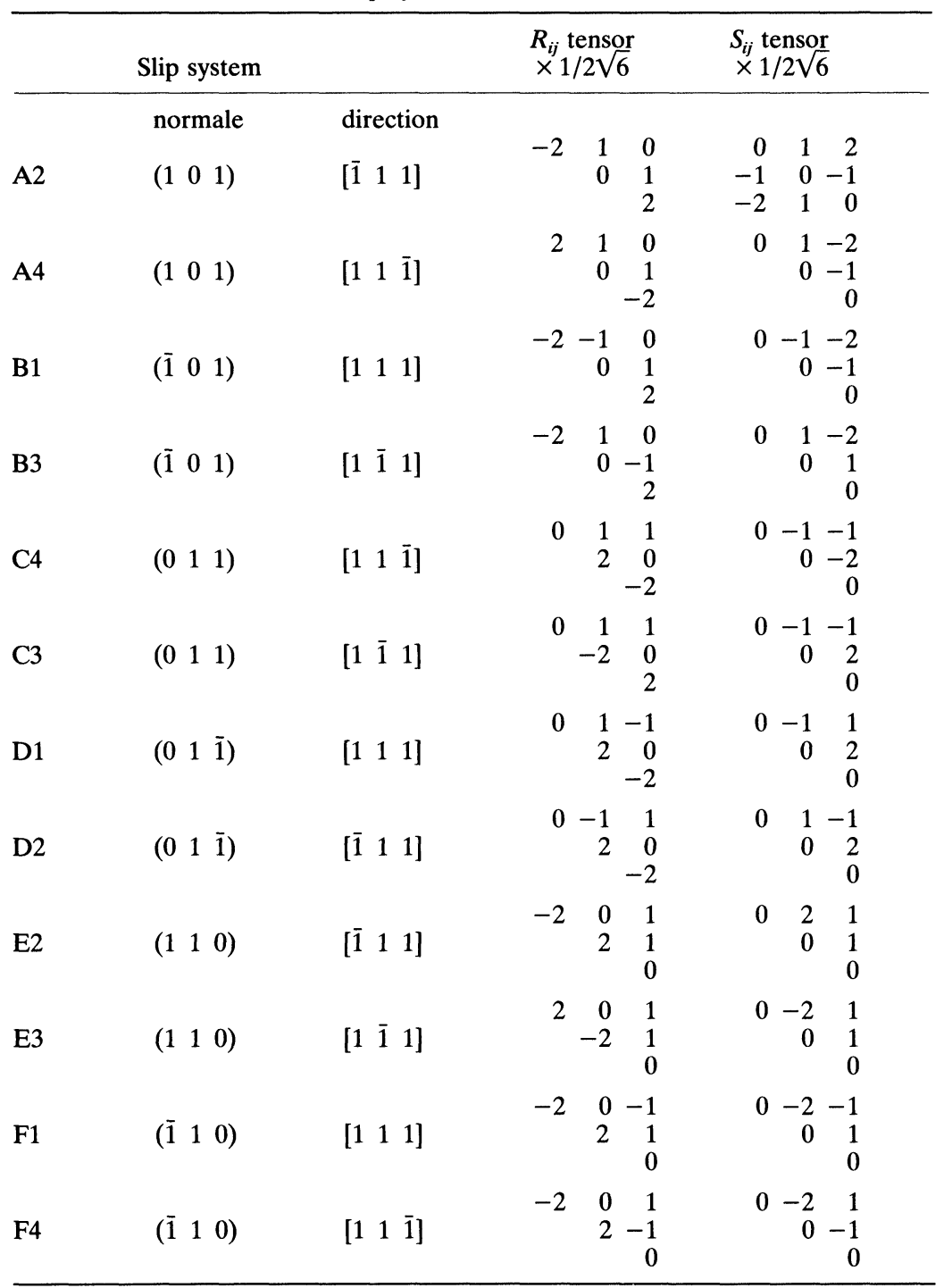


(a)

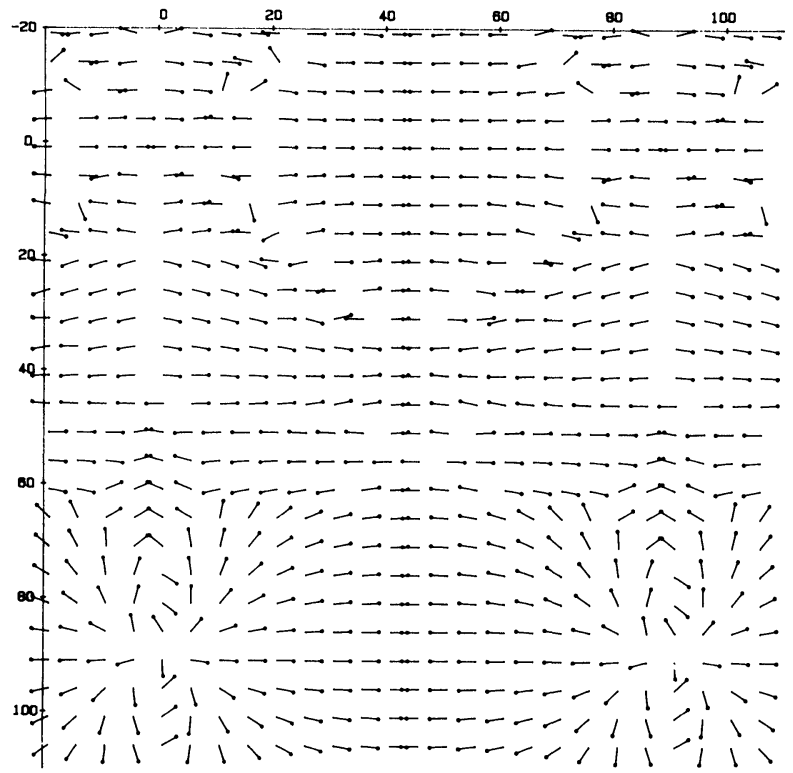

(b)

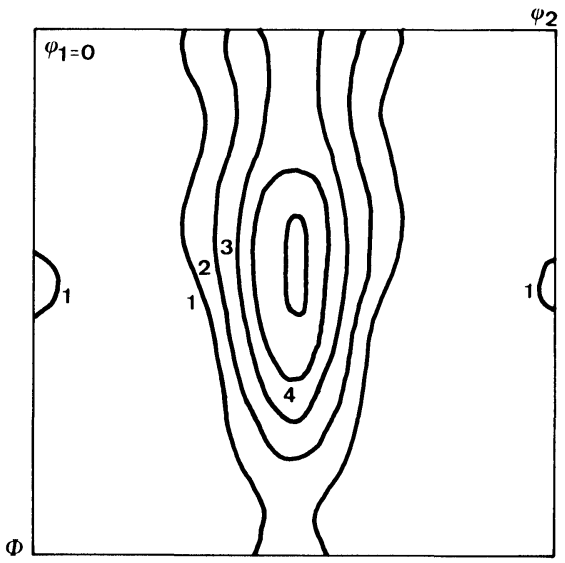

Figure 8 Rotation field of B.C.C. crystalline lattices in $\varphi_{1}=0^{\circ}$ plane: (a) calculated texture using lower stability criterion, (b) experimental texture measured by Bunge (1968). 
(a)

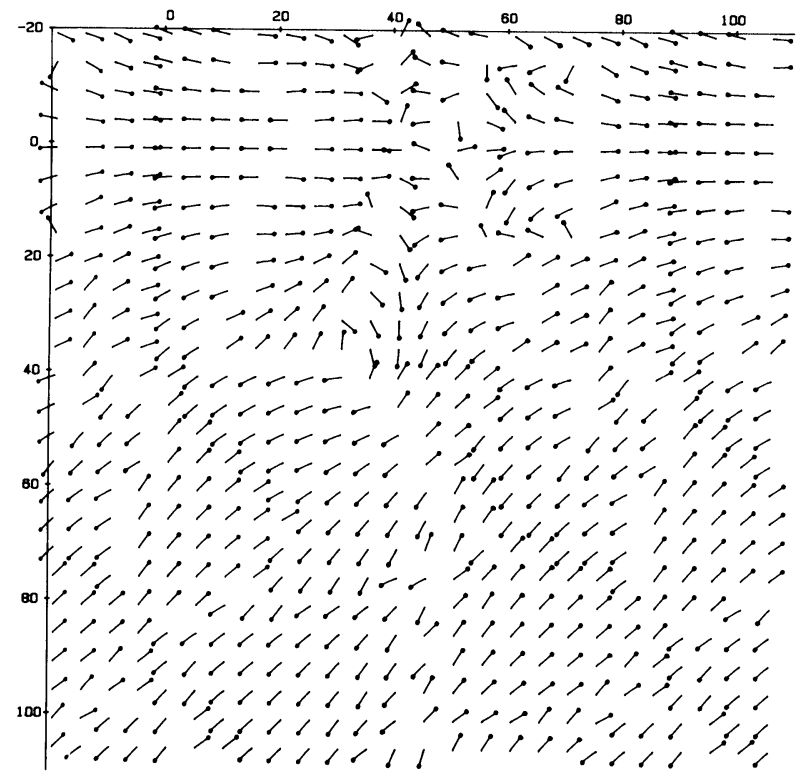

(b)

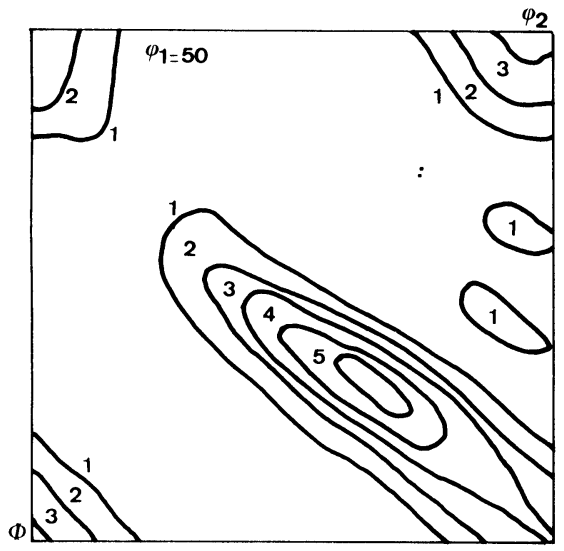

Figure 9 Rotation field of B.C.C. crystalline lattices in $\varphi_{1}=50^{\circ}$ plane: (a) calculated texture using lower stability criterion, (b) experimental texture measured by Bunge (1968). 
(a)

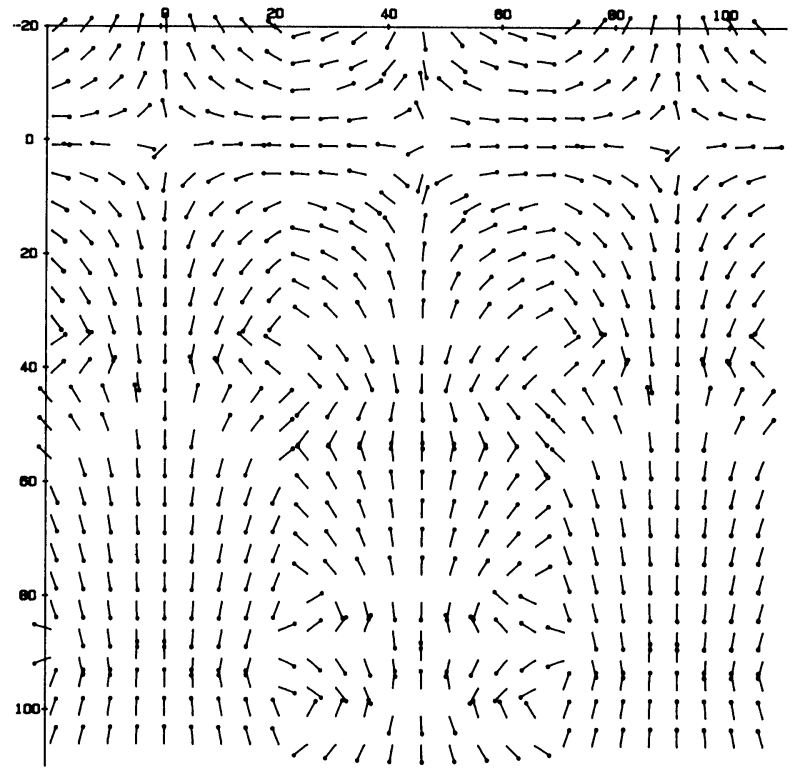

(b)

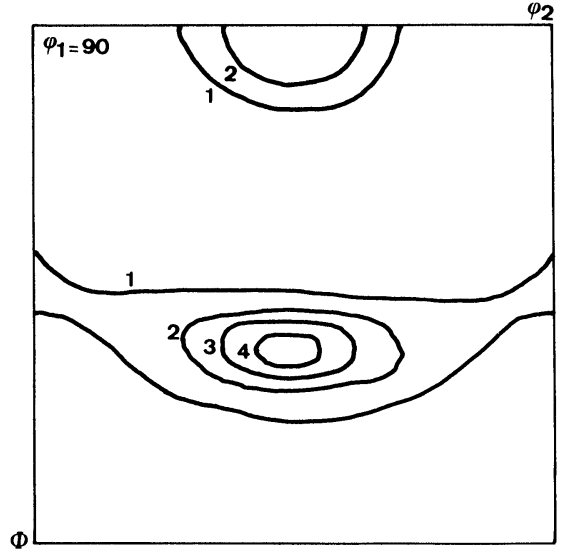

Figure 10 Rotation field of B.C.C. crystalline lattices in $\varphi_{1}=90^{\circ}$ plane: (a) calculated texture using lower stability criterion, (b) experimental texture measured by Bunge (1968). 


\section{DISCUSSION AND CONCLUSIONS}

The new concept of plastic deformation modes is introduced in this study. Two deformation modes are essentially distinguished; the homogeneous multislip and the single slip. They are considered independently on other deformation mechanisms such as twinning or slip on grain boundaries. The competition between these two deformation modes strongly depends on the latent hardening. In general, the homogeneous multislip is favoured by a weak latent hardening. Even if the latent hardening is important, this deformation mode may take place at the regions of strong stress concentration such as grain boundaries or triple nodes. In these zones, the primary slip introduces internal stresses of such amplitude that the homogeneous multislip may take place. The extent of these zones depends on the latent hardening. Outside of the regions of stress concentration, the single slip deformation mode is predominant when the latent hardening is important. If the idea of two deformation modes is adopted, the question of compatibility between these two regions arises. Factors such as particular microstructure, grain shape, properties of grain boundaries have been neglected. This compatibility is obtained by means of a particular plastic strain inhomogeneity.

Two regions of constant plastic strains separated by so called habit plane are supposed to exist. The consequences of this partition of the plastic strain of a polycrystal into two deformation modes, as a function of latent hardening, are numerous. In this study only the influence of the partition on the formation of strain induced textures has been discussed in details.

1. The particular texture is associated with each of the deformation mode.

-homogeneous multislip produces preferentially the copper-type texture.

-single slip induces more often brass-type texture.

The contribution of various texture components depends on volume fraction of each deformation mode, which in turn may be correlated with the latent hardening and also with grain size. When the grain diameter is small, the homogeneous multislip may occupy almost all 
grain volume. In this case, the copper-type texture is observed even if the latent hardening is important. A similar indication was mentioned by Lin and Ahlers (1973). They observed that the increase of grain size leads to an increase of the brass-component contribution for the case of transition textures.

2 . The second consequence is closely related with the creation of habit planes during the plastic flow of polycrystal. This phenomenon confers a particular microstructure to the polycrystal, which is superposed on its granular structure. We have shown that the repartition of orientations of habit planes is not random but coincides with the orientation of shear bands.

Relation between the formation of habit planes and the development of microshear bands and their coalescence in macrobands may be suggested. An analysis of this mechanism is undertaken and constitutes the object of another study. The superposition of the initial or current granular structure and the microstructure due to the oriented habit planes confers a "long range organisation" to the polycrystal caused by correlation of orientations of the habit planes. In order to describe the behavior of such a material, the use of more complex statistical methods, such as that recently developed by Lipinski and Berveiller for elastoplastic materials, is necessary.

\section{APPENDIX I: SOLUTION SATISFYING THE PRINCIPLE OF MINIMAL PLASTIC WORK RATE}

The equations of Chapter II define a class of kinematically admissible fields related to some particular deformation modes. The whole twelve slip systems of F.C.C. or B.C.C. metals can satisfy the conditions established in Chapter II, but the real solution, belonging to the kinematically admissible class must be chosen to minimize the plastic work rate:

$$
d W=\int_{V} \sigma_{i j}(r) \dot{\varepsilon}_{i j}^{p}(r) d V
$$

Dividing by the total volume $V$ and adding over all grains, this 
expression becomes:

$$
d W=\frac{1}{V} \sum_{I} \int_{V I} \sigma_{i j}(r) \dot{\varepsilon}_{i j}^{p}(r) d V
$$

or

$$
\sum_{I}\left\{\frac{1}{V} \int_{V M} \sigma_{i j}^{M} \dot{E}_{i j}^{p} d V+\frac{1}{V} \int_{V^{I \alpha}} \sigma_{i j}^{I} \dot{\varepsilon}_{i j}^{p I} d V\right\}
$$

where $V M$ is the matrix volume and $V^{I \alpha}$ the single slip zone volume in the grain $I$.

Using the volume fractions, we have:

$$
d W=f \sigma_{i j}^{M} \dot{E}_{i j}^{p}+\sum_{I} f^{I} \sigma_{i j}^{I} R_{i j}^{I k} \dot{\gamma}^{I}
$$

the product $\sigma_{i j}^{I} R_{i j}^{I k}$ is the reduced shear stress $\tau^{k}$ (equal to critical shear stress $\tau_{c}^{k}$ for the real strain field).

Supposing (like in the Taylor model), the initial critical shear stress to be the same for all slip systems, we can write:

$$
d W=f \sigma_{i j}^{M} \dot{E}_{i j}^{p}+\tau_{c} \sum_{I} f^{I}\left|\dot{\gamma}^{I}\right|
$$

For one given strain rate field, this quantity is minimal if the second term is minimal. Consequently, in each grain, for the same volume fraction $f^{I}$, the minimum is obtained for the minimal absolute value of $\dot{\gamma}$. Otherwise, among the twelve kinematically admissible solutions corresponding to the twelve slip systems, the retained solution is that of minimal $|\dot{\gamma}|$. This principle is analogous to that established by Taylor and being the basis of all Taylor-type models.

\section{APPENDIX II: RELATIVE STABILITY OF DOUBLE SOLUTION OF EQN. (13)}

In order to establish the theory of Chapter II, a piece-wise plastic strain field due to a plane interface (in the mathematical sense) has been supposed. This interface supports a superficial dislocation density associated with the first order compatibility relation without a long range stress field which is cancelled using the second order compatibility conditions. In fact, the interface possesses some finite 
"thickness" due to local interactions between interfacial dislocations. These last ones induce a local stress field caused by the dipolar part of superficial dislocation density. The analysis of these incompatibilities (in the sense of the second order incompatibility condition) is treated in another paper by the authors where it is shown that the local incompatibility tensor contains two parts $\eta^{1}$ and $\eta^{2}$ such that:

$$
\begin{aligned}
& \eta_{i j}^{1}(u)=\varepsilon_{i k m} \varepsilon_{j l n} N_{k} N_{l} D_{m n}\left\{\delta^{\prime}(u-h)+\delta^{\prime}(u)\right\} \\
& \eta_{i j}^{2}(u)=\varepsilon_{i k m} \varepsilon_{j l n} N_{k} N_{l} S_{m n}\left\{\delta^{\prime}(u-h)-\delta^{\prime}(u)\right\}
\end{aligned}
$$

where:

$$
\begin{aligned}
D_{m n} & =\frac{1}{2}\left(\varepsilon_{m n}^{p I}-E_{m n}^{p}\right) \\
S_{m n} & =\frac{1}{2}\left(\varepsilon_{m n}^{p I}+E_{m n}^{p}\right)
\end{aligned}
$$

and $\vec{N}$ is the unit normal vector to the considered habit plane. $h$ is the "physical" thickness of the habit plane, and $u$ means the distance from a given point to the habit plane. $\delta(u)$ is the Dirac distribution function and $\delta^{\prime}(u)$ its derivative with respect to $u$.

We recognize that the compatibility condition (11) can only be satisfied for $\eta_{i j}^{1}=0$, but $\eta_{i j}^{2}$ disappears only if $h=0$, which is not realistic for cold work. The two habit planes with normal vectors $\vec{N}_{1}$ and $\vec{N}_{2}$ respectively obtained from condition $\eta_{i j}^{1}=0$, lead to residual incompatibilities $\eta_{i j}^{2}\left(\vec{N}_{1}\right)$ and $\eta_{i j}^{2}\left(\vec{N}_{2}\right)$ which are different and consequently, generate different stress fields.

Without performing the complete calculation of these stresses (which is done by Lipinski et al., to be published), we compare only the norms $\left|\eta^{2}\left(\vec{N}_{1}\right)\right|^{2}=\eta_{i j}^{2}\left(\vec{N}_{1}\right) \eta_{i j}^{2}\left(\vec{N}_{1}\right)$ and $\left|\eta^{2}\left(\vec{N}_{2}\right)\right|^{2}=\eta_{i j}^{2}\left(\vec{N}_{2}\right) \eta_{i j}^{2}\left(\vec{N}_{2}\right)$. It enables us to furnish a quantitative indication concerning the elastic energy associated to these stresses. Thus, the habit plane with lower elastic energy is called the stable one.

This analysis is used to separate the lattice rotation fields into two parts, but this separation does not mean that one of these fields should be eliminated (see Lipinski et al., to be published).

\section{References}

Berveiller, M. (1980) Sciences et Techniques de l'Armement, t 54, 521-619.

Berveiller, M. and Bouaouine H. (1987) Arch. of Met. 32(2) 171-191.

Berveiller, M., Zaoui, A. (1979) J. Mec. Phys. Solids 26, 325-344. 
Bunge, H. J., Mathematische Methoden der Texturanalyse, Akademie Verlag, Berlin (1969).

Bunge, H. J. (1968) Phys. Stat. Sol. 26, 167-172.

Dillamore, I. L., Roberts, W. T., Acta Met. 12 (1964), 281-293.

Fargette, B., Whitwham, D., (1976) Mem. Sci. Rev. F. Metall Mars 198-206.

Franciosi, P., Berveiller, M., Zaoui, A., (1980) Acta Met. 28, 273-283.

Haasen, P., Phil. Mag. (1958) 3, 384-389.

Hirsch, J., Virnich, K., Lücke, K., (1981) ICOTOM 6,(1) 375-384.

Honnef, H., Mecking, H. (1978) Proc. 5th Int. Conf. Texture of Materials Ed. C. Gottstein, K. Lücke Springer 265-275.

Jaoul, B., (1965). Etude de la plasticité et application aux métaux Ed. Dunod.

Kröner, E., (1958) Kontinuums Theorie der Versetzungen und Eigenspannungen, Berlin, Springer Verlag.

Kröner, E., (1961) Acta Met. 155-161.

Korbel, A. and Martin, P. (1986) Acta Met. 34, 1905-1909.

Korbel, A. and Martin, P. (To be published).

Korbel, A., Embury, J. D., Hatherly, M., Martin, P. L. and Erbsloh, H. W. (1986) Acta Met. 34(10) 1999-2009.

Leffers, T. (1969) Zeit Metallkunde 60, 785-786.

Liu, Y. C. and Ahlers, G. A. (1973) Met. Trans. 4, 1491-1497.

Lipinski, P. and Berveiller, M. (To appear in Int. J. Plasticity).

Lipinski, P., Bouaouine, H. and Berveiller, M. (To be published).

Meissner, J. (1959) Z. Metallkunde 50, 207-217.

Mussot, P., (1983). Thèse 3e cycle Université Paris VI.

Mughrabi, H. (1983) Acta Met. 31, 1367-1379.

Rasmussen, K. V. and Pedersen, O. B., (1980) Acta Met. 1467-1478.

Rey, C. and Zaoui, A., (1982) Acta Met. 30, 523-535.

Sachs, G., (1928) Zeitschrift VDI 72, 734-736.

Smallman, R. E., Green, P., (1964) Acta Met. 12, 145-154.

Wassermann, G., (1963) Zeit. Metallkunde 54, 61-65.

Wassermann, G., Heye, W., (1968), Zeit. Metallkunde 59, 693-697.

Winter, A. T., (1974) Phil. Mag. 30, 719-738.

Taylor, G. I., (1938) J. Inst. Metals 62, 307-324.

Truszkowski, W. and Krol, J., (1968) J., Mem. Sci. Rev. Met. 65, 907.

Van Houtte, P., (1984) ICOTOM 7, 7-23. 